\title{
Positive Affect and Sleep in Spousal Alzheimer Caregivers:
}

\section{A Longitudinal Study}

Short title: Positive Affect and Sleep in Dementia Caregivers

\author{
${ }^{1,2,3}$ Roland von Känel MD \\ ${ }^{3}$ Brent T. Mausbach, PhD \\ ${ }^{3,4}$ Sonia Ancoli-Israel, $\mathrm{PhD}$ \\ ${ }^{3}$ Paul J. Mills, PhD \\ ${ }^{3}$ Joel E. Dimsdale, MD \\ ${ }^{3}$ Thomas L. Patterson, PhD \\ ${ }^{3}$ Igor Grant, MD
}

${ }^{1}$ Division of Psychosomatic Medicine, Department of General Internal Medicine, Inselspital, Bern University Hospital and University of Bern, Switzerland

${ }^{2}$ Department of Clinical Research, University of Bern, Switzerland

${ }^{3}$ Department of Psychiatry, University of California San Diego, La Jolla, CA

${ }^{4}$ Department of Medicine, University of California San Diego, La Jolla, CA

\section{Corresponding author:}

Roland von Känel, MD; Department of General Internal Medicine; Division of Psychosomatic Medicine; Bern University Hospital; Inselspital; CH-3010 Bern; Switzerland Tel.: +41 3163220 19; fax: +41 3138211 84; e-mail: roland.vonkaenel@insel.ch 


\begin{abstract}
We examined the longitudinal relationship between positive affect (PA) and sleep in 126 spousal Alzheimer's disease caregivers. Caregivers underwent four yearly assessments for the Positive and Negative Affect Schedule, the self-rated Pittsburgh Sleep Quality Index, and actigraphy to objectify nighttime total sleep time, wake after sleep onset and percent sleep. Increased levels of PA and a greater positivity (i.e., positive-to-negative affect) ratio were significantly associated with better subjective sleep over the entire study period. Yearly increases in PA -even when controlling for negative affect (NA)- and in the positivity ratio also were associated with better subjective sleep. PA and actigraphy measures showed no significant relationship. Increased PA is longitudinally associated with better sleep in dementia caregivers largely independent of NA.
\end{abstract}




\section{INTRODUCTION}

Compared to non-caregiving controls, caregivers of a family member with

Alzheimer's disease (AD) have poor subjective sleep as can be measured by the Pittsburgh Sleep Quality Index (PSQI) (Wilcox, \& King, 1999; von Känel et al., 2006). In terms of objective sleep, cross-sectional studies with in-home polysomnography found shorter total sleep time (TST) (von Känel et al., 2006), lower sleep efficiency (von Känel et al., 2006), and less time spent in restorative sleep stages (Fonareva, Amen, Zajdel, Ellingson, \& Oken, 2011) in $\mathrm{AD}$ caregivers compared to non-caregiving controls. Actigraphy studies to assess sleepwake-activity revealed greater wake after sleep onset (WASO) and less sleep percent in AD caregivers relative to controls (von Känel et al., 2010). Much of the variance in poor subjective sleep, but not in objective sleep of dementia caregivers is explained by negative affect (NA) and stress, both in cross-sectional (McCurry, Logsdon, Teri, \& Vitiello, 2007; Kochar, Fredman, Stone, \& Cauley, 2007; Rowe, McCrae, Campbell, Benito, \& Cheng, 2008) and longitudinal studies (von Känel et al, 2012).

Positive affect (PA) such as happiness, cheerfulness, and enjoyment is an important dimension of good mental health (Headey, Kelley, \& Wearing, 1993); PA also is associated with good physical health (Steptoe, Wardle, \& Marmot, 2005) and longevity in healthy and diseased populations (Chida, \& Steptoe, 2008). Some investigators propose that PA and NA systems operate independently from each other (Cacioppo, \& Berntson, 1994), even though PA and NA show a modest inverse relationship (Russell, \& Carroll, 1999), Therefore, when probing for beneficial effects of PA on health outcomes, it seems advisable to test for PA alone as well as for PA controlling at the same time for NA (Chida, \& Steptoe, 2008). In addition, a ratio of perceived PA-to-NA - the so-called "positivity ratio" - of about 3:1 has been discussed to be particularly associated with flourishing mental health (Fredrickson, \& Losada, 2005). In other words, across several studies, an optimal level of emotional well- 
being and positive mental health is achieved if individuals experience three times more PA than NA (Diehl, Hay, \& Berg, 2011). This conceptualization agrees with the World Health Organization (2004) defining mental health not with the absence of mental illness alone but the presence of a state of well-being that allows a person to cope with life stress and to make contributions to the community. Compared to adulthood, even higher positivity ratios and thus emotional well-being can be expected in older adult populations as the preponderance of PA to NA increases across the lifespan (Diehl, Hay, \& Berg, 2011).

Spousal AD caregivers endorse significantly lower levels of PA than non-caregivers (Adams, 2008; Mausbach, Chattillion, Roepke, Patterson, \& Grant, 2013). In older noncaregiving adults from the community, daily associations were found between more subjective sleep complaints (but not sleep actigraphy measures) and lower PA (without adjustment for NA) over a study period of 14 days (McCrae et al., 2008). However, to our knowledge, the association between PA and sleep has not previously been investigated in elderly dementia caregivers (McCurry, Logsdon, Teri, \& Vitiello, 2007; McCurry, Gibbons, Logsdon, Vitiello, \& Teri, 2009). The identification of potentially modifiable contributors to poor caregiver sleep such as PA is important, as this knowledge might inform tailored interventions aimed at improving the poor mental and physical health in dementia caregivers (McCurry, Logsdon, Teri, \& Vitiello, 2007).

Conceptually, it follows from the above literature that the relationship of PA to sleep can be modeled either by considering PA alone, or by accounting as well for NA. In this study we modeled these relationships in community-dwelling spousal AD caregivers in three ways: model 1 investigated the longitudinal relationship of PA to sleep variables (self report and actigraphy); model 2 adjusted for NA; and model 3 utilized the ratio of PA to NA (positivity ratio). We predicted these relationships to be independent of other important demographic and health-related correlates of AD caregiver's sleep. 


\section{METHODS}

\section{Study participants}

The participants of this study comprised the total sample of 126 caregivers who had been enrolled in the University of California, San Diego (UCSD) Alzheimer's Caregiver Study, which investigates mechanisms potentially linking stress of spousal AD caregivers with poor mental and physical health. The UCSD Institutional Review Board approved the study protocol. All participants provided written consent. Caregivers were recruited from local support groups, through referrals from local caregiver agencies, from community health fairs, and through referrals from other participants. To be eligible, caregivers were required to be at least 55 years old, married, and dwelling in the community with their spouse. They had to provide primary care for a spouse with a physician-based diagnosis of AD. Exclusion criteria were presence of any major illnesses (e.g., cancer), severe hypertension, or treatment with steroids, non-selective beta-blockers, or oral anticoagulants.

\section{Study design}

The study applied a longitudinal design in which all participants underwent annual inhome assessments by trained research staff for a period of up to 4 years (one caregiver also contributed an assessment after 5 years). A total of 48 caregivers were not recruited until year 2 or 3 of the project. Therefore and because the study with its follow-up had to be terminated early due to lack of funding, not all caregivers were able to complete all 4 years of follow-up. During each assessment, participants provided relevant sociodemographic data, physical and mental health characteristics, and actigraphy measures. Moreover, research staff encouraged caregivers to call in should there be a major transition in the relationship with their spouse (i.e., placement or bereavement). Participation in the study was not terminated if the care recipient transited to a long-term care facility or deceased. However, in the case of these two 
transitions, an assessment appointment was scheduled approximately 3 months after a transition. By design, post-transition assessments occurred at 3, 15, 27, and 39 months. These post-transition assessments replaced the annual assessments. For example, if a caregiver had a regular assessment at 12 months (i.e., the year 1 follow-up), and the following year had a placement at 11 months (i.e., at this time being 23 months in the study), he or she had an assessment at 26 months (i.e., the year 2 follow-up), which assessment replaced the 24 months assessment. The next assessment (i.e. the year 3 follow-up) was 12 months later (i.e., the caregiver being 38 months in the study at this time).

\section{Sociodemographic data and medical history}

We collected information on gender, age, and years of education to define socioeconomic status. To assess the number of health problems, we asked caregivers the following question "Do you currently have, or has a doctor told you that you have, any of the following health problems?" and provided a list with 18 items (e.g., arthritis, heart disease, high blood pressure, problems with your kidneys, psychiatric problems). The number of positive items was summed to reflect medical comorbidity. Participants also indicated whether they had used antidepressant drugs over the past 30 days (yes/no).

\section{Health behaviors}

We asked participants for their weight and height to calculate body mass index (BMI). Smoking status was defined as ever smokers (i.e., all former plus a few current smokers) vs. never smokers. We used the Rapid Assessment of Physical Activity scale to assess the amount of light, moderate, and strenuous physical activities in a typical week, including strength and flexibility exercises (total score 0-6) (Topolski et al., 2006). We scored the amount of consumed alcohol in the past month based on the number of days subjects had at 
least one alcoholic drink and the number of alcoholic drinks they usually drank on these days.

\section{Caregiver stress and affect}

We obtained information on years caregiving, role overload, and problem behaviors of the care recipient. Role overload with life responsibilities was rated using a 4 item scale (Pearlin, Mullan, Semple, \& Skaff, 1990) which was shown to reflect a valid construct in AD caregivers (Mausbach et al., 2012b); for example "you work hard as a caregiver but never seem to make any progress." We used average responses to each item $(1=$ not at all, $4=$ completely) to create a total overload score (range 1-4). Cronbach's $\alpha$ for the current sample was 0.76 for the baseline assessment.

We used the Revised Memory and Behavior Problem Checklist to assess 24 behavioral problems ( 7 memory, 9 disruptive, and 8 depressive) endorsed by the AD spouse during the past week; this instrument is recommended as a valid tool for the empirical assessment of behavior problems in dementia patients (Teri et al., 1992). Typical items relate to whether the spouse loses or misplaces things, appears sad or depressed, or becomes aggressive to others verbally. Coefficient $\alpha$ for the total scale was 0.81 for the current sample at baseline. We used the Positive and Negative Affect Scale to assess NA and PA in the last few weeks (Watson, Clark, \& Tellegen, 1988). The PANAS has shown its validity in older

adults (Ready et al., 2011). Caregivers rated 10 items for NA (e.g., irritable, nervous, hostile) and PA (e.g., excited, proud, active) each on a 5-point scale (1=very slightly or not at all, 5= extremely; total score 10-50 for either scale). Coefficient $\alpha$ was 0.88 for the PA scale and 0.84 for the NA scale for the current sample at baseline. We also calculated the positivity ratio by dividing the total score from the PA items by the total score from the NA items. 


\section{Sleep assessment}

We used the interviewer-administered Pittsburgh Sleep Quality Index (PSQI) to assess self-rated sleep during the past month (Buysse, Reynolds, Monk, Berman, \& Kupfer, 1989). In terms of its validity for the current study, the PSQI has previously been applied to healthy subjects over 80 years (Buysse et al., 1991). The PSQI comprises 19 items, which can be grouped along seven component scores, which are subjective sleep quality, sleep duration, sleep latency, sleep disturbances, sleep efficiency, use of sleep medication and daytime dysfunction. The seven component scores are summed up to yield a global PSQI score ranging from 0 to 21 points. Higher scores indicate poorer self-rated sleep quality, whereby pathological difficulties with sleep are reflected by a global score greater than 5 . The overall coefficient $\alpha$ for the seven PSQI components was 0.68 for the baseline assessment. After completing the PSQI caregivers started actigraphy on the same day.

Actigraphy was used to assess objective sleep in terms of nighttime TST, nighttime WASO, and nighttime sleep percent (i.e., the percent of minutes scored as sleep). Caregivers wore the SleepWatch-O actigraph (Ambulatory Monitoring, Inc., Ardsley, NY) on the nondominant wrist for three consecutive 24 -hour periods (i.e. 72 hours). The actigraph data represent averages of the three consecutive night periods. The device detects movement via a piezoelectric bimorph-ceramic cantilever that generates a voltage each time the actigraph is moved. Voltages are gathered continuously and summarized over one-minute intervals. We report data based on digital proportional integration (PIM) mode (Motionlogger User's Guide), a modality that is preferably applied to older adults (Blackwell et al., 2008). ActionW-2 software (Ambulatory Monitoring, Inc., Ardsley, NY) was used to analyze actigraphy data (Action-W User's Guide). Caregivers also completed a sleep log to record bed and wake times as well as intervals of actigraph removals for particular activities (e.g. 
showering). This information was utilized in editing the actigraph records as previously described (Blackwell et al., 2005).

\section{Data Analysis}

Data were analyzed with PASW 18.0 statistical software package (SPSS Inc., Chicago, IL) with two-tailed significance at $p \leq 0.05$. Non-parametric tests were used to compare groups on baseline characteristics and to estimate correlation coefficients between variables. We conducted a mixed (random-effects) regression analysis to examine the covariate-adjusted relationship between PA and sleep measures (i.e., PSQI, TST, WASO, sleep percent). Mixed model regression allows one to estimate an intercept and slope for each participant based on the available data for that individual (i.e., even when some points are missing across assessments), augmented by the data from the entire sample (Singer, \& Willett, 2003). We selected covariates a-priori based on previous research showing that these may affect dementia caregivers' sleep and thus might confound the relation between PA and sleep: age, gender, socioeconomic status, caregiver health (including body mass index and antidepressant medication) and health behaviors (i.e., smoking, physical activity, alcohol consumption), caregiving-related stress (e.g., role overload and problem behaviors of the care recipient), and major transitions in the caregiving situation (McCurry, Logsdon, Teri, \& Vitiello, 2007; McCurry, Gibbons, Logsdon, Vitiello, \& Teri, 2009; von Känel et al., 2012).

The model included the following fixed effects: age at baseline, gender, education, years caregiving, placement status of the AD spouse (yes/no), deceased status of the AD spouse (yes/no), health problems, antidepressant use (yes/no), BMI, ever smoking (yes/no), physical activity, alcohol consumption, role overload, and problem behaviors. Of these, years caregiving, placement and deceased status of the $\mathrm{AD}$ spouse, health problems, antidepressant use, BMI, smoking status, physical activity, alcohol consumption, role overload and problem 
behaviors were all entered as time-varying. Random intercepts were modelled for participants. The effect of PA was tested in three ways. Model 1 treated PA as the predicting variable of sleep; model 2 tested for PA as the predicting variable after controlling for NA; model 3 tested for the positivity ratio (i.e., PA scores divided by NA scores) as the predicting variable.

We computed between- and within-person effects of PA on sleep. Between-caregiver effects were computed as the deviation of each caregiver's overall mean PA level (for model 1 and model 2), mean NA level (for model 2), and mean positivity ratio (for model 3) across all his/her observations from the grand mean of these measures. The between-caregiver analysis allows one to answer the question whether caregivers who are generally (i.e., over the entire study period) high in PA are also those reporting better sleep. We only computed grand mean values for variables with hypothesized relationships. Within-caregiver effects were computed by centering PA scores, NA scores, and the positivity ratio around each caregiver's personal mean. That is, each subject's personal mean value across time was subtracted from each of his/her yearly observations. The within-caregiver analysis allows one to answer the question whether at individual assessments a caregiver who reports a high level of PA also has good sleep, thereby suggesting that there is an association between yearly fluctuations in PA and sleep. Note that if between- and within-caregiver effects were both significant, this would mean that a constant level of PA over the entire study period is significantly related to sleep but also that changes (up or down) in PA and sleep are significantly related to each other from one assessment to the next. 


\section{RESULTS}

\section{Characteristics of caregivers}

Number of assessments: Table 1 shows the characteristics of study participants at baseline and for the subsequent yearly assessments. The 126 included caregivers contributed 500 assessments across all assessments corresponding to a mean of 4.0 assessments per caregiver. Data for all of the fixed effect and time-variant variables were complete in $100 \%$ of these 500 assessments except for years caregiving (complete in 98.6\%) and for PA and NA (complete in $99.6 \%$ each). Sleep measures were available in $100 \%$ for the PSQI and in $92.0 \%$ for each actigraphy measure.

Data at baseline: At enrollment into the study, caregivers had been providing care for their AD spouse for an average of 4.3 years (range $0.5-17.1$ ). The positivity ratio (i.e., the ratio of PA to NA) was $\geq 3$ in $17.5 \%$ of caregivers and $57.1 \%$ had a PSQI score $>5$. Higher PA was associated with lower NA $(r=-0.30, p=0.001)$ and greater physical activity $(r=0.18$, $\mathrm{p}=0.040)$. In addition PA was higher in men than in women (33.6 \pm 7.1 vs. $31.0 \pm 7.5, p=0.050)$ and in ever smokers than never smokers $(32.8 \pm 7.5$ vs. $30.9 \pm 7.3, \mathrm{p}=0.048)$. With respect to associations with sleep measures, PA showed an inverse relation with the PSQI ( $\mathrm{r}=-0.22$, $\mathrm{p}=0.015$ ), but no relation with any actigraphy measure (all $\mathrm{p}$-values $>0.15$ ). PA also was unrelated to age, education, years caregiving, health problems, antidepressant use, BMI, alcohol consumption, role overload and care recipient problem behaviors (all p-values $>0.06$ ). In terms of intercorrelations between sleep measures, the PSQI showed no significant correlations with any actigraphy measure (all p-values $>0.13$ ). In contrast, there were expected correlations between TST and sleep percent $(\mathrm{r}=0.35, \mathrm{p}<0.001)$ and between WASO and sleep percent $(\mathrm{r}=-0.93, \mathrm{p}<0.001)$. 


\section{Longitudinal relationship between positive affect and sleep}

Tables 2 and 3 show the fully adjusted models for the associations of PA with subjective and objective sleep for the entire study period.

\section{Between-caregiver effects}

Subjective sleep quality: Table 2 shows that caregivers with higher mean levels of PA over the study period had significantly better subjective sleep $(\mathrm{p}=0.016)$ (model 1). However, when additionally controlling for NA, the relationship between PA and PSQI became nonsignificant $(\mathrm{p}=0.25)$, while greater mean level of NA was significantly associated with lower subjective sleep quality over the study period $(\mathrm{p}=0.001)$ (model 2$)$. A greater mean level of the positivity ratio over the study period also was associated with a significantly better subjective sleep quality $(\mathrm{p}<0.001)($ model 3$)$.

In all three models for the PSQI, elevated PSQI scores (i.e., decreased mean subjective sleep quality) over the study period was significantly predicted by female gender, increased role overload, more problem behaviors, and deceased status of the AD spouse (all p-values $<0.015)$.

Objective sleep quality: As can be seen in Table 2, there were no significant relationships between any PA measure and objective measures of TST, WASO, or sleep percent (models 1-3). NA also was not significantly associated with any actigraphy measure. In terms of significant sleep correlates, more health problems were related to shorter TST and use of antidepressants was related to longer TST over time. Greater BMI and deceased status of the AD spouse were both associated with greater WASO and reduced sleep percent. More problem behaviors endorsed by the care recipient also was associated with greater WASO. 


\section{Within-caregiver effects}

Subjective sleep quality: Table 3 shows that increases in PA from one assessment to another was associated with significant improvement in subjective sleep quality $(\mathrm{p}<0.001)$ (model 1). This effect was maintained after additionally controlling for NA $(\mathrm{p}=0.002)$, whereby NA emerged as a predictor of poor sleep quality on its own ( $\mathrm{p}=0.018)(\operatorname{model} 2)$. Caregivers with an increase in the positivity ratio between two assessments also experienced improvement in subjective sleep quality during the same time interval ( $\mathrm{p}=0.001)$ (model 3). As in Table 2, female gender, increased role overload, more problem behaviors, and deceased status of the AD spouse also were significantly associated with the PSQI (data not shown).

Objective sleep quality: Table 3 further reveals that changes in PA measures across individual assessments were not significantly associated with TST, WASO, or sleep percent (models 1-3). A similar finding emerged for NA in model 2. The same covariates as seen in Table 2 showed significant associations with actigraphy measures (data not shown).

\section{DISCUSSION}

We investigated the longitudinal association of PA, PA adjusted for NA, and the positivity ratio (i.e., levels of PA divided by levels of NA) with both subjective and objective measures of sleep in elderly caregivers of a spouse with AD. The main finding from our study was that increased levels of PA were associated with reports of better sleep in spousal AD caregivers. This relationship was independent of a range of sociodemographic, behavioral, medical, and also psychological covariates of poor self-rated sleep. We adjusted our analyses for caregiver-specific subjective and objective burden, namely duration of caregiving, major transitions in the caregiver situation, perceived role overload, and problem behaviors of the AD spouse (Schulz, \& Martire, 2004). As in our previous study (von Känel et al., 2012) that had a shorter follow-up than the current investigation, we found elevated 
role overload and death of the AD spouse to be associated with poor subjective sleep. Concurrent with our observation, various problem behaviors of the dementia patient, particularly night-time awakenings, might also affect caregivers' sleep perception (McCurry et al., 1999).

We found PA to be related to subjective sleep both over the entire study period (i.e., between-caregiver effects) as well as when testing for associations between yearly fluctuations in PA and subjective sleep (i.e., within-caregiver effects). The latter finding concurs with a previous study in older adults who were not caregivers, showing that withinperson change of PA and NA were associated with respectively better and poorer subjective sleep quality on a daily base over two weeks; however, that study did not adjust for the mutual effects of PA and NA (McCrae et al., 2008). We found that with additional adjustment for NA, the between-caregiver relationship of PA with the PSQI became nonsignificant. In contrast, NA became a significant predictor to suggest that poor subjective sleep over the entire study period was more related to high levels of NA than to low levels of PA. However, the concurrent change seen in PA and subjective sleep from one yearly assessment to the next was independent of NA. Taken together, this might imply that the perception of good subjective sleep is more responsive to shorter-term (i.e., yearly) fluctuations in PA than to sustained perception of high PA. In other words, once NA was taken into account, caregivers could have high PA over the entire study period but what really was related to subjective sleep was their increase or decrease in PA levels.

Another way of looking at PA effects on sleep is by testing for the positivity ratio. Although PA and NA are viewed as two independent affect systems, a person usually endorses both PA and NA, although their relative amounts may differ at times. Accordingly, it has been shown that a higher ratio of the amount of PA divided by the amount of NA is associated with better health in older adults (Diehl, Hay, \& Berg, 2011), with a ratio of 3:1 
viewed as being critical for mental flourishing by some authors (Fredrickson, \& Losada, 2005). We found that for one point increase in the positivity ratio, there was an approximate decrease in the mean total PSQI score of 1.3 points over time and of 0.7 points for the yearly assessments. These effects may seem of clinical relevance as they were of similar size as gender and deceased status of the AD spouse. Moreover, the average global PSQI score over time was 6.5 , which is above the cut-off of 5 indicative of pathological sleep. Our results suggest that an increase of 1 point in the positivity ratio would be expected to yield on average almost normal subjective sleep in our caregiver sample. Nevertheless, it remains unclear whether caregivers with only a slightly elevated PSQI score would show relevant sleep improvement with an increase in PA.

In contrast to the associations with subjective sleep, we did not find evidence for the hypothesis that PA was significantly related to objective actigraphic measures of sleep. This concurs with a recent study in a convenience sample of older adults in whom daily assessments of PA and subjective sleep complaints showed an inverse relationship, while PA was unrelated to actigraphy data (McCrae et al., 2008). It also has been shown that caregiver burden, depressive symptoms, and other forms of NA explain much of the variance in selfreported sleep, while actigraphy is less influenced by depression (Rowe, McCrae, Campbell, Benito, \& Cheng, 2008). The available data together with those from the present study suggest that, like NA domains, the PA domains also show stronger associations with subjective sleep than with objective sleep.

Our findings might help to broaden the understanding of the mechanisms linking low PA, disturbed sleep and poor physical health in dementia caregivers. Specifically, compared to their non-caregiving counterparts, dementia caregivers endorse less PA (Adams, 2008; Mausbach, Chattillion, Roepke, Patterson, \& Grant, 2013) and they also run a higher risk of developing cardiometabolic disturbances (von Känel et al., 2011) and coronary heart disease 
(CHD) (von Känel et al., 2008). We recently showed low leisure satisfaction of AD caregivers to be associated with reduced endothelial function, which plays a critical role in atherosclerosis (Mausbach et al., 2012a). In contrast, longitudinal studies reveal a reduced risk of incident CHD in individuals with greater amount of PA (Kubzansky, \& Thurston, 2007). The PSQI global score integrates several aspects of poor self-reported sleep, including insomnia complaints, sleep duration, and use of sleeping pills (Buysse et al., 2007). A recent meta-analysis of 13 prospective studies found poor subjective sleep defined as difficulties of initiating or maintaining sleep, and restless/disturbed nights to be associated with a 1.5-fold increased relative risk of developing or dying from cardiovascular disease (Sofi et al., in press). In elderly subjects, the PSQI and sleep duration $\leq 5 \mathrm{~h}$ both showed a direct association with intima-media thickness suggesting poor subjective sleep and short sleep both are associated with subclinical atherosclerosis (Nakazaki et al., 2012). Interestingly, prescribed sleeping pill users show excess mortality, even after controlling for reported sleep duration and insomnia (Kripke, Garfinkel, Wingard, Klauber, \& Marler, 2002). It follows from these studies, that the relation of low PA with poor subjective sleep might critically compromise cardiovascular health in dementia caregivers. Our data would suggest that (yearly) fluctuations in PA as they relate to poor subjective sleep might be more relevant for cardiovascular health than constant levels of PA, although this needs to be studied further. Behavioral activation programs have the potential to increase the engagement of distressed and activity restricted dementia caregivers in pleasurable activities so to increase their PA levels (Mazzucchelli, Kane, \& Rees, 2010; Mausbach et al., 2011). It thus seems warranted to test whether dementia caregivers' cardiovascular health might benefit from improvement in subjective sleep that is achieved by an increase in PA through behavioral activation techniques. Our findings suggest that shorter-term increase in PA and decrease in NA, respectively, will both be associated with improved subjective sleep, but once PA levels 
remain constant, intervening on the level of NA might be a more promising approach to improve subjective sleep.

Our study has its strengths and limitations. An average of four yearly assessments allowed us to compute both between-subject and within-subject effects which to some extent yielded differential information. The ample count of total assessments yielded enough power to reliably adjust for a number of important covariates of sleep. However, another informative way of looking at the data would have been to compute correlations between trajectories of change in PA and sleep measures, but due to the dramatic fall-off of data points at year 4 , such an analysis would have suffered from insufficient statistical power. We judge the high drop out of caregivers for the year 4 assessment to largely be at random because attrition was due to lack of funding and therefore not based on subject factors. This is important because non-random attrition might have hampered internal validity of our findings. We did not apply in-home polysomnography that might have yielded more accurate objective sleep data than actigraphy. Actigraphy however, gave us the ability to collect three nights of data rather than one night of PSG. Although we applied a longitudinal design, we cannot disentangle the direction of the relationship between PA and sleep, which likely is bidirectional. The PSQI only covers the previous 30 days of self-reported sleep, which can be problematic when attempting to answer questions about the sleep/affect relationship across a 4-year period. We adjusted our analysis for measures reflecting mental health status, including caregiver distress, antidepressant use, and "psychiatric problems" as one possible comorbidity. However, we did not perform a formal psychiatric interview to assess mental health status, specifically depression. Compared to their non-caregiving counterparts, AD caregivers have elevated levels of depressive mood (Mausbach, Chattilion, Roepke, Patterson, \& Grant, 2013) which, in turn, relate to poor subjective sleep (von Känel et al., 2012). Because the concepts of NA and depression are not independent, as evidenced by their 
high correlation ( $\mathrm{r}=0.71$ in our sample at baseline; details not shown) we did not adjust our analyses of NA for depressed mood so as to avoid multicollinearity effects.

To sum up, elevated levels of PA are longitudinally associated with better subjective sleep but not objective sleep in AD caregivers. This relationship is partially independent of NA. Whether behavioral interventions to increase PA may also improve dementia caregivers' sleep and ultimately caregiver health remains to be seen.

\section{ACKNOWLEDGMENTS}

The authors are grateful to Susan Calleran and Christine Gonzaga for data collection. This study was supported by the National Institutes of Health/National Institute on Aging through award AG 15301 to Igor Grant. Additional support was provided through award AG 03090 to Brent Mausbach and AG 08415 to Sonia Ancoli-Israel.

\section{REFERENCES}

Action-W User's Guide, Version 2.0. Ardsley, New York.

Adams, K.B. (2008). Specific effects of caring for a spouse with dementia: differences in depressive symptoms between caregiver and non-caregiver spouses. International Psychogeriatrics, 20, 508-520.

Blackwell, T., Ancoli-Israel, S., Gehrman, P.R., Schneider, J.L., Pedula, K.L., \& Stone, K.L. (2005). Actigraphy scoring reliability in the study of osteoporotic fractures. Sleep, 28, 1599-1605.

Blackwell, T., Redline, S., Ancoli-Israel, S., Schneider, J.L., Surovec, S., Johnson, N.L., Cauley, J.A., Stone, K.L; Study of Osteoporotic Fractures Research Group. (2008). Comparison of sleep parameters from actigraphy and polysomnography in older women: the SOF study. Sleep, 31, 283-291. 
Buysse, D.J., Reynolds, C.F. 3rd., Monk, T.H., Berman, S.R., \& Kupfer, D.J. (1989). The Pittsburgh Sleep Quality Index: a new instrument for psychiatric practice and research. Psychiatry Research, 28, 193-213.

Buysse, D.J., Reynolds, C.F. $3^{\text {rd }}$., Monk, T.H., Hoch, C.C., Yeager, A.L., Kupfer, D. J (1991). Quantification of subjective sleep quality in healthy elderly men and women using the Pittsburgh Sleep Quality Index (PSQI). Sleep, 14, 331-338.

Buysse, D.J., Thompson, W., Scott, J., Franzen, P.L., Germain, A., Hall, M., Moul, D.E., Nofzinger, E.A., Kupfer, D.J. (2007). Daytime symptoms in primary insomnia: a prospective analysis using ecological momentary assessment. Sleep Medicine, 8, 198208.

Cacioppo, J.T., \& Bernston, G.G. (1994). Relationship between attitudes and evaluative space: A critical review with emphasis on the separability of positive and negative substrates. Psychological Bulletin, 115, 401-423

Chida, Y., \& Steptoe, A. (2008). Positive psychological well-being and mortality: a quantitative review of prospective observational studies. Psychosomatic Medicine, 70, 741-756.

Diehl, M., Hay, E.L., \& Berg, K.M. (2011). The ratio between positive and negative affect and flourishing mental health across adulthood. Aging \& Mental Health, 15, 882-893.

Fonareva, I., Amen, A.M., Zajdel, D.P., Ellingson, R.M., Oken, B.S. (2011). Assessing sleep architecture in dementia caregivers at home using an ambulatory polysomnographic system. Journal of Geriatric Psychiatry and Neurology, 24, 50-59.

Fredrickson, B.L., \& Losada, M.F. (2005). Positive affect and the complex dynamics of human flourishing. American Psychologist, 60, 678-686.

Headey, B., Kelley, J., \& Wearing, A. (1993). Dimensions of mental health: Life satisfaction, positive affect, anxiety and depression. Social Indicators Research, 29, 63-82. 
Kochar, J., Fredman, L., Stone, K.L., Cauley, J.A., \& Study of Osteoporotic Fractures. (2007). Sleep problems in elderly women caregivers depend on the level of depressive symptoms: results of the Caregiver--Study of Osteoporotic Fractures. Journal of the American Geriatrics Society, 55, 2003-2009.

Kripke, D.F., Garfinkel, L., Wingard, D.L., Klauber, M.R., \& Marler, M.R. (2002). Mortality associated with sleep duration and insomnia. Archives of General Psychiatry, 59, 131136.

Kubzansky, L.D., \& Thurston, R.C. (2007). Emotional vitality and incident coronary heart disease. Archives of General Psychiatry, 64, 1393-1401.

Mausbach, B.T., Chattillion, E.A., Moore, R.C., Roepke, S.K., Depp, C.A., \& Roesch, S. (2011). Activity restriction and depression in medical patients and their caregivers: a meta-analysis. Clinical Psychology Review, 31, 900-908.

Mausbach B.T., Chattillion, E., Roepke, S.K., Ziegler, M.G., Milic, M., von Känel, R., Dimsdale, J.E., Mills, P.J., Patterson, T.L., Allison, M.A., Ancoli-Israel, S., \& Grant, I. (2012a). A longitudinal analysis of the relations among stress, depressive symptoms, leisure satisfaction, and endothelial function in caregivers. Health Psychology, 31, 433440.

Mausbach, B.T., Roepke, S.K., Chattillion, E.A., Harmell, A.L., Moore, R, Romero-Moreno, R., Bowie, C.R., \& Grant, I. (2012b). Multiple mediators of the relation between caregiving stress and depressive symptoms. Aging \& Mental Health, 15, 27-38.

Mausbach, B.T., Chattillion, E.A., Roepke, S.K., Patterson, T., \& Grant, I. (2013). A comparison of psychosocial outcomes in elderly Alzheimer caregivers and noncaregivers. American Journal of Geriatric Psychiatry, 21, 5-13.

Mazzucchelli, T.G., Kane, R.T., \& Rees, C.S. (2010). Behavioral activation interventions for well being: A meta-analysis. Journal of Positive Psychology, 5, 105-121. 
McCrae, C.S., McNamara, J.P., Rowe, M.A., Dzierzewski, J.M., Dirk, J., Marsiske, M., \& Craggs, J.G. (2008). Sleep and affect in older adults: using multilevel modeling to examine daily associations. Journal of Sleep Research, 17, 42-53.

McCurry, S.M., Logsdon, R.G., Teri, L., \& Vitiello, M.V. (2007). Sleep disturbances in caregivers of persons with dementia: contributing factors and treatment implications. Sleep Medicine Reviews, 11, 143-153.

McCurry, S.M., Gibbons, L.E., Logsdon, R.G., Vitiello, M.V., \& Teri, L. (2009). Insomnia in caregivers of persons with dementia: who is at risk and what can be done about it? Sleep Medicine Clinics, 4, 519-526.

McCurry, S.M., Logsdon, R.G., Teri, L., Gibbons, L.E., Kukull, W.A., Bowen, J.D., McCormick, W.C., \& Larson, E.B. (1999). Characteristics of sleep disturbance in community-dwelling Alzheimer's disease patients. Journal of Geriatric Psychiatry and Neurology, 12, 53-59.

McKibbin, C.L., Ancoli-Israel, S., Dimsdale, J., Archuleta, C., von Känel, R., Mills, P., Patterson, T. L., \& Grant, I. (2005). Sleep in spousal caregivers of people with Alzheimer's disease. Sleep, 28, 1245-1250.

Motionlogger User's Guide: Act Milennium. Ardsley, New York.

Nakazaki, C., Noda, A., Koike, Y., Yamada, S., Murohara, T., \& Ozaki, N. (2012).

Association of insomnia and short sleep duration with atherosclerosis risk in the elderly. American Journal of Hypertension, 25, 1149-1155.

Pearlin, L.I., Mullan, J.T., Semple, S.J., \& Skaff, M.M. (1990). Caregiving and the stress process: an overview of concepts and their measures. The Gerontologist, 30, 583-594.

Ready, R.E., Vaidya, J.G., Watson, D., Latzman, R.D., Koffel, E.A., \& Clark, L.A. (2011). Age-group differences in facets of positive and negative affect. Aging \& Mental Health, 15, 784-795. 
Rowe, M.A., McCrae, C.S., Campbell, J.M., Benito, A.P., \& Cheng, J. (2008). Sleep pattern differences between older adult dementia caregivers and older adult noncaregivers using objective and subjective measures. Journal of Clinical Sleep Medicine, 4, 362-369.

Russell, J.A., \& Carroll, J.M. (1999). On the bipolarity of positive and negative affect. Psychological Bulletin, 125, 3-30.

Schulz, R., \& Martire, L.M. (2004). Family caregiving of persons with dementia: prevalence, health effects, and support strategies. American Journal of Geriatric Psychiatry, 12, 240249.

Singer, J.D., \& Willett, J.B. (2003). Applied longitudinal data analysis: modelling change and event occurrence. New York: Oxford University Press.

Sofi, F., Cesari, F., Casini, A., Macchi, C., Abbate, R., \& Gensini, G.F. (in press). Insomnia and risk of cardiovascular disease: a meta-analysis. European Journal of Preventive Cardiology.

Steptoe, A., Wardle, J., \& Marmot, M. (2005). Positive affect and health-related neuroendocrine, cardiovascular, and inflammatory processes. Proceedings of the National Academy of Sciences of the United States of America, 102, 6508-6512.

Teri, L., Truax, P., Logsdon, R., Uomoto, J., Zarit, S., \& Vitaliano, P.P. (1992). Assessment of behavioral problems in dementia: The revised memory and behavior problems checklist. Psychology and Aging, 7, 622-631.

Topolski, T.D., LoGerfo, J., Patrick, D.L., Williams, B., Walwick, J., \& Patrick, M.B. (2006). The Rapid Assessment of Physical Activity (RAPA) among older adults. Preventing Chronic Disease, 3, A118.

von Känel, R., Dimsdale, J.E., Ancoli-Israel, S., Mills, P.J., Patterson, T.L., McKibbin, C.L., Archuleta, C., \& Grant, I. (2006). Poor sleep is associated with higher plasma proinflammatory cytokine interleukin-6 and procoagulant marker fibrin D-dimer in older 
caregivers of people with Alzheimer's disease. Journal of the American Geriatrics Society, 54, 431-437.

von Känel, R., Mausbach, B.T., Patterson, T.L., Dimsdale, J.E., Aschbacher, K., Mills, P.J., Ziegler, M.G., Ancoli-Israel, S., \& Grant I. (2008). Increased Framingham Coronary Heart Disease Risk Score in dementia caregivers relative to non-caregiving controls. Gerontology, 54, 131-137.

von Känel, R., Ancoli-Israel, S., Dimsdale, J.E., Mills, P.J., Mausbach, B.T., Ziegler, M.G., Patterson, T.L., \& Grant, I. (2010). Sleep and biomarkers of atherosclerosis in elderly Alzheimer caregivers and controls. Gerontology, 56, 41-50.

von Känel, R., Mausbach, B.T., Dimsdale, J.E., Mills, P. J., Patterson, T.L., Ancoli-Israel, S., Ziegler, M.G., Roepke, S.K., Chattillion, E.A., Allison, M., \& Grant, I. (2011). Cardiometabolic effects in caregivers of nursing home placement and death of their spouse with Alzheimer's disease. Journal of the American Geriatrics Society, 59, $2037-$ 2044.

von Känel, R., Mausbach, B.T., Ancoli-Israel, S., Dimsdale, J.E., Mills, P.J., Patterson, T.L., Ziegler, M.G., Roepke, S.K., Chattillion, E.A., Allison, M., \& Grant, I. (2012). Sleep in spousal Alzheimer caregivers: a longitudinal study with a focus on the effects of major patient transitions on sleep. Sleep, 35, 247-255.

Watson, D., Clark, L.A., \& Tellegen, A. (1988). Development and validation of brief measures of positive and negative affect: The PANAS scales. Journal of Personality and Social Psychology, 54, 1063-1070.

Wilcox, S., \& King, A.C. (1999). Sleep complaints in older women who are family caregivers. The Journals of Gerontology Series B: Psychological Sciences and Social Sciences, 54, P189-P198. 
World Health Organisation. (2004). Promoting mental health: Concepts, emerging evidence, practice (Summary report). Geneva. 
Table 1. Characteristics of the sample at baseline and yearly assessments

\begin{tabular}{|c|c|c|c|c|c|}
\hline Variables & Baseline (n=126) & Year $1(n=115)$ & Year $2(n=109)$ & Year $3(n=100)$ & Year $4(n=49)$ \\
\hline Age (years) & $74.2 \pm 7.9$ & $75.4 \pm 7.8$ & $76.4 \pm 7.9$ & $77.7 \pm 7.9$ & $78.8 \pm 7.6$ \\
\hline Women $(\%)$ & 70.6 & 69.6 & 71.6 & 69.0 & 69.4 \\
\hline Education (years) & $15.2 \pm 3.0$ & $15.1 \pm 3.1$ & $15.1 \pm 3.1$ & $15.1 \pm 3.1$ & $15.3 \pm 3.3$ \\
\hline Years caregiving & $4.33 \pm 3.38$ & $5.39 \pm 3.52$ & $6.37 \pm 3.52$ & $7.34 \pm 3.39$ & $8.54 \pm 3.44$ \\
\hline Spouse placed (\%) & 0 & 13.0 & 28.4 & 39.0 & 51.0 \\
\hline Spouse deceased $(\%)$ & 0 & 6.1 & 18.3 & 33.0 & 59.2 \\
\hline Antidepressant drug (\%) & 26.2 & 27.0 & 26.6 & 22.0 & 20.4 \\
\hline Body mass index $\left(\mathrm{kg} / \mathrm{m}^{2}\right)$ & $26.5 \pm 4.7$ & $26.8 \pm 5.2$ & $26.4 \pm 5.1$ & $27.0 \pm 5.1$ & $25.9 \pm 3.9$ \\
\hline Ever smoker $(\%)$ & 46.0 & 46.1 & 44.0 & 48.0 & 49.0 \\
\hline Physical activity (score) & $3.42 \pm 1.64$ & $3.10 \pm 1.37$ & $3.04 \pm 1.73$ & $2.80 \pm 1.61$ & $3.47 \pm 1.50$ \\
\hline Alcohol consumption (score) & $5.60 \pm 5.82$ & $5.87 \pm 6.67$ & $6.08 \pm 6.40$ & $5.99 \pm 6.75$ & $6.82 \pm 6.98$ \\
\hline Positive affect (score) & $31.8 \pm 7.4$ & $31.1 \pm 7.1$ & $31.9 \pm 7.7$ & $32.2 \pm 7.4$ & $33.1 \pm 6.8$ \\
\hline Positivity ratio & $2.00 \pm 0.90$ & $2.14 \pm 0.90$ & $2.36 \pm 1.05$ & $2.31 \pm 0.99$ & $2.46 \pm 1.01$ \\
\hline Pittsburgh sleep quality index & $6.69 \pm 3.57$ & $6.26 \pm 3.55$ & $6.54 \pm 3.31$ & $6.09 \pm 3.83$ & $5.93 \pm 3.46$ \\
\hline Actigraphy total sleep time (min) & $438 \pm 68$ & $442 \pm 68$ & $434 \pm 63$ & $425 \pm 61$ & $436 \pm 66$ \\
\hline Actigraphy wake after sleep onset (min) & $63.8 \pm 28.6$ & $64.8 \pm 34.6$ & $60.5 \pm 27.6$ & $64.0 \pm 33.3$ & $60.2 \pm 25.1$ \\
\hline Actigraphy sleep percent (\%) & $87.3 \pm 5.4$ & $87.3 \pm 6.1$ & $87.8 \pm 5.4$ & $87.0 \pm 6.2$ & $87.8 \pm 5.1$ \\
\hline
\end{tabular}


Values are given as means \pm SD or percentages. Note that a given caregiver could have experienced no transition in his or her caregiving situation, long-term placement of the spouse only, death of the spouse only, or placement with subsequent death of the spouse; in the latter case, this caregiver contributed a transition to both the "spouse placed" and the "spouse deceased" category. 
Table 2. Between-caregiver effects for the relations between positive affect and sleep measures across the entire study period

\begin{tabular}{|c|c|c|c|c|c|c|c|c|}
\hline \multirow[b]{2}{*}{ Model 1} & \multicolumn{2}{|c|}{$\begin{array}{l}\text { Pittsburgh Sleep } \\
\text { Quality Index (score) }\end{array}$} & \multicolumn{2}{|c|}{$\begin{array}{l}\text { Actigraphy total sleep } \\
\text { time (min) }\end{array}$} & \multicolumn{2}{|c|}{$\begin{array}{l}\text { Actigraphy wake after } \\
\text { sleep onset (min) }\end{array}$} & \multicolumn{2}{|c|}{$\begin{array}{l}\text { Actigraphy sleep } \\
\text { percent }(\%)\end{array}$} \\
\hline & Estimate & S.E. & Estimate & S.E. & Estimate & S.E. & Estimate & S.E. \\
\hline Female gender & 1.433 & $0.610^{*}$ & & & & & & \\
\hline Spouse deceased & 0.993 & $0.402 *$ & & & 15.379 & $4.596 * *$ & -2.635 & $0.823 * *$ \\
\hline Health problems & & & -4.122 & $1.827 *$ & & & & \\
\hline Antidepressant drug & & & 20.269 & $7.773 * *$ & & & & \\
\hline Body mass index & & & & & 0.899 & $0.431^{*}$ & -0.171 & $0.081 *$ \\
\hline Role overload & 0.230 & $0.050 * * *$ & & & & & & \\
\hline Problem behaviors & 0.098 & $0.031 * *$ & & & 0.879 & $0.354 *$ & & \\
\hline Positive affect between & -0.102 & $0.042 *$ & -0.937 & 0.812 & 0.380 & 0.360 & -0.099 & 0.070 \\
\hline \multicolumn{9}{|l|}{ Model 2} \\
\hline Female gender & 1.237 & $0.588^{*}$ & & & & & & \\
\hline Spouse deceased & 0.999 & $0.400 *$ & & & 15.419 & $4.600 * *$ & -2.638 & $0.824 * *$ \\
\hline Health problems & & & -4.133 & $1.829 *$ & & & & \\
\hline Antidepressant drug & & & 20.475 & $7.791 * *$ & & & & \\
\hline Body mass index & & & & & 0.925 & $0.437 *$ & -0.174 & $0.082 *$ \\
\hline Role overload & 0.203 & $0.051 * * *$ & & & & & & \\
\hline Problem behaviors & 0.095 & $0.031 * *$ & & & 0.887 & $0.355^{*}$ & & \\
\hline Negative affect between & 0.184 & $0.055^{* *}$ & 0.622 & 1.115 & -0.213 & 0.493 & 0.023 & 0.095 \\
\hline Positive affect between & -0.050 & 0.043 & -1.116 & 0.879 & 0.321 & 0.388 & -0.093 & 0.075 \\
\hline \multicolumn{9}{|l|}{ Model 3} \\
\hline Female gender & 1.202 & $0.587 *$ & & & & & & \\
\hline Spouse deceased & 0.997 & $0.400^{*}$ & & & 15.331 & $4.600 * *$ & -2.620 & $0.824 * *$ \\
\hline Health problems & & & -3.919 & $1.822 *$ & & & & \\
\hline Antidepressant drug & & & 21.099 & $7.776 * *$ & & & & \\
\hline Body mass index & & & & & 0.912 & $0.433 *$ & -0.173 & $0.081 *$ \\
\hline Role overload & 0.205 & $0.051 * * *$ & & & & & & \\
\hline Problem behaviors & 0.096 & $0.310 * *$ & & & 0.904 & $0.354 *$ & -0.127 & $0.063 *$ \\
\hline Positivity ratio between & -1.265 & $0.299 * * *$ & -1.967 & 6.094 & 1.733 & 2.683 & -0.388 & 0.521 \\
\hline
\end{tabular}


Three separate models were tested: Model 1 treated positive affect (PA) as the predicting variable of sleep; Model 2 tested for PA as the predicting variable with adjustment for negative affect (NA); Model 3 tested for the positivity ratio (i.e., PA scores divided by NA scores) as the predicting variable.

Data are given as slopes (B), ${ }^{*} \mathrm{p}<0.050, * * \mathrm{p}<0.010 ; * * * \mathrm{p}<0.001$. Only significant covariates are shown. Covariates in all models: age (baseline), gender, years of education, years caregiving, years in study, caregiver transitions (spouse placed, spouse deceased), number of caregiver health problems, antidepressant drug use (yes/no), body mass index, ever smoking (yes/no), physical activity, alcohol consumption, role overload, number of care recipient problem behaviors. 
Table 3. Within-caregiver effects for the relations between positive affect and sleep measures across the entire study period

\begin{tabular}{|c|c|c|c|c|c|c|c|c|}
\hline \multirow[b]{2}{*}{ Model 1} & \multicolumn{2}{|c|}{$\begin{array}{l}\text { Pittsburgh Sleep } \\
\text { Quality Index (score) }\end{array}$} & \multicolumn{2}{|c|}{$\begin{array}{l}\text { Actigraphy total sleep } \\
\text { time (min) }\end{array}$} & \multicolumn{2}{|c|}{$\begin{array}{l}\text { Actigraphy wake after } \\
\text { sleep onset (min) }\end{array}$} & \multicolumn{2}{|c|}{$\begin{array}{l}\text { Actigraphy sleep } \\
\text { percent }(\%)\end{array}$} \\
\hline & Estimate & S.E. & Estimate & S.E. & Estimate & S.E. & Estimate & S.E. \\
\hline Positive affect within & -0.091 & $0.026 * * *$ & -0.917 & 0.812 & -0.336 & 0.311 & 0.037 & 0.055 \\
\hline \multicolumn{9}{|l|}{ Model 2} \\
\hline Negative affect within & 0.065 & $0.027 *$ & -0.355 & 1.115 & -0.158 & 0.337 & 0.027 & 0.060 \\
\hline Positive affect within & -0.079 & $0.026 * *$ & -0.982 & 0.599 & -0.366 & 0.317 & 0.042 & 0.056 \\
\hline \multicolumn{9}{|l|}{ Model 3} \\
\hline Positivity ratio within & -0.715 & $0.213 * *$ & -3.954 & 4.949 & 2.747 & 2.600 & 0.348 & 0.461 \\
\hline
\end{tabular}

Three separate models were tested: Model 1 treated positive affect (PA) within as the predicting variable of sleep; Model 2 tested for PA within as the predicting variable with adjustment for negative affect (NA) within; Model 3 tested for the positivity ratio (i.e., PA within scores divided by NA within scores) as the predicting variable.

Data are given as slopes $(\mathrm{B}),{ }^{*} \mathrm{p}<0.05, * * \mathrm{p}<0.01 ; * * * \mathrm{p}<0.001$. Covariates in all models: age (baseline), gender, years of education, years caregiving, years in study, caregiver transitions (spouse placed, spouse deceased), number of caregiver health problems, antidepressant drug use 
(yes/no), body mass index, ever smoking (yes/no), physical activity, alcohol consumption, role overload, number of care recipient problem behaviors. No significant correlates of sleep measures are shown as they were the same as those in Table 2 . 